\title{
Wireless Sensor Network Routing Protocols: A Survey
}

\author{
Parul Khurana \\ Panjab University \\ Chandigarh, India
}

\author{
Inderdeep Aulakh \\ Panjab University \\ Chandigarh, India
}

\begin{abstract}
Advancements in wireless sensor networks (WSN) over the past two decades has foreseen an increased interest in the potential use in applications like combat field, security surveillance, border protection, disaster management and reconnaissance. Moreover, researchers have termed the 21st century as the "Sensor Decade" [3]. Sensor nodes are remotely deployed in large numbers and expected to operate autonomously in harsh environments. Now-a-days routing protocols are being designed, where energy awareness is an essential designing issue. In this paper, survey on recent routing protocols for sensor networks along with their classification and approaches is done . Each routing protocol is described under the appropriate category followed by possible future research areas.
\end{abstract}

\section{Keywords}

WSN, hierarchical routing, QoS, location routing, bio-inspired routing

\section{INTRODUCTION}

WSN is an emerging information acquisition technology integrating the latest technological advancements in microelectronics and network communications. It forms an intelligent network application system consisting of thousands of tiny energy limited and low cost nodes. Sensor nodes autonomously sense, process and communicate via the radio medium forming a self-organized network pattern. Typically, sensors report sensed data to an external base station for further processing. They are equipped with low-cost and small-capacity batteries which are mostly non-rechargeable and irreplaceable. Due to decrement in size and cost of sensors, the possible use of a large set of disposable unattended sensors has increased. Thus, motivating the intensive research for addressing the potential of collaboration among sensors in data gathering, processing, coordinating and managing the data flow and sensing activity. Large numbers of tiny sensor nodes help to obtain data about physical phenomena in an easy way in comparison to the conventional methods adopted. Sensor network routing is a challenging task. This is due to several characteristics that distinguish them from wireless ad hoc networks and contemporary communication. Since every application challenge and quality factors are different, so there is a need for diversified routing techniques. This survey is made to understand some of the wireless sensor network applications, their routing algorithms and the QOS parameters for the application domain. Although there are some previous efforts for surveying the communication protocols their applications and characteristics in WSNs [1]. The scope of the survey presented in this paper is distinguished from these surveys in many aspects. In Section 3, a comprehensive survey of routing techniques in WSNs is done which is followed by a summary of future research directions on routing in Section 4.

\section{RELATED WORK}

Emergence of new architectural schemes has inspired the efforts for surveying the communication protocols and their characteristics along with applications for wireless sensor networks [1][13]. The goal of [1] is to make a comprehensive survey of features and challenges for sensor network routing which describes the constraints on sensor nodes and the protocols proposed for energy efficient routing. A survey of applications on sensor networks focuses on QOS needs and routing protocols which satisfy the QOS needs is also studied[2]. It is a broad classification of protocols of WSN . Although a variety of routing protocols are covered [1][2], but the paper does not specify complete taxonomy for such routing protocols. Thereby not giving the complete scope of the survey. Thus, for this reason the survey in this paper is more focused. It serves deeper insight for routing techniques in wireless sensor networks along with their unique features. Moreover, the research here includes a comprehensive list of recently proposed routing protocols. In [13] typical sensor node architecture is considered along with its components. Here, distinct architectural factors(like network dynamics and data delivery model) and attributes of sensor networks are described. This helps in selecting the right application for infrastructure used. However, the paper does not describe any routing protocol and the potential effects of infrastructure design on route setup. Therefore, in this paper focus is on the network layer protocols(responsible for route discovery and maintenance) that categorizes different approaches for data routing.

\section{ROUTING PROTOCOLS IN WIRELESS SENSOR NETWORKS}

Routing protocols have a wide scope in research area when implemented in WSN. These protocols can be classified on the basis of network structure (designed for the application), routing paths established, and network operations and as an initiator of communications. Fig 1 shows the taxonomy of routing protocols which are further sub-divided into subcategories [11] and helpful in designing of network protocol. In this section, the routing protocols for WSNs are discussed.

\subsection{Network Structure Protocols}

Routing protocols depending on network structure based routing protocols behaves significantly with respect to the design constraints given for the network structure or area. These are in turn classified as Flat routing(Data Centric), hierarchical routing (Clustering) and Location (Geographic) routing.

\subsubsection{Flat Routing(Data Centric)}

In Data Centric Routing, data is transmitted to every sensor node within the deployment region with significant redundancy. This is due to absence of global identifiers through which selection of specific sensor nodes to be queried 
is not feasible. Here, every sensor node plays the same roles such as data dissemination and communication with the sink [11]. Sink sends queries to some regions and then wait for data from the sensors located in the selected regions to facilitate data-centric characteristics

\section{a) Flooding and Gossiping}

The most traditional network routing are flooding and gossiping [4]. In flooding, every sensor node receives a data packet which in turn broadcasts it to all the neighboring nodes. As soon as the packet reaches the destination or the maximum number of hops is reached, the broadcasting process stops. Though flooding is easy but it has several drawbacks like overlap, resource blindness and implosion problem. The problem of implosion is avoided by gossiping. Unlike classical broadcasting which sends packets to all neighbors, it sends information to a random neighbor. As a result, only the selected neighbors will forward the packet to the destination. Also it does not require routing tables and topology maintenance.

\section{b) SPIN}

SPIN (Sensor Protocol for Information via Negotiation) [4] is an adaptive data centric communication protocol that disseminates information from sensors in an energy constrained WSN. It overcomes the implosion and overlap problem of classic flooding. Sensor nodes consult with each other by sending meta-data before they actually transmit data i.e. they negotiate among themselves. Negotiation ensures the elimination of redundant data and transfer of useful information takes place. Thus, these nodes need to monitor their own energy resources and avoid its excess use. When transferring the data to its target SPIN is not sure if data would reach successfully which becomes its disadvantage. Also it does not perform well in the high-density distribution of nodes. Another drawback of SPIN [5] is that if the nodes interested in the data are distant from the source node and the nodes between source and target are not interested in that data, then data will not be delivered to the target. Thus data blind spots would appear which will affect the information collection of the network and not making it a good choice for applicationns.

\section{c) Rumor Routing}

Rumor Routing is a variation of directed diffusion. It routes the queries to the nodes that observes a particular event and maintains only one path from source to sink [6]. On the detection of an event, it is added to a local table called events table. Here, it generates a long lived packet called an agent. The propagation of information about local events to distant nodes is done by agents. Then the visited nodes tables are combined with their own event table on the way. Whenever an agent crosses a path which leads to another event, an aggregate path is created. If a shorter path is found by an agent than its own it updates the shorter path immediately i.e. by inspecting the requisite event table. It is useful for applications that has small number of events as the communication cost is also reduced. Also it helps in avoiding flooding in SPIN.

\section{d) Directed Diffusion}

Directed Diffusion(DD) is a data-centric application-aware routing protocol used for collecting and publishing information in wireless sensor networks. DD has been developed to fulfill the requirement of data flowing from sink to sensors [7]. Gradients are introduced in DD to search the intermediate nodes for the matching data in a particular direction. The process of diffusion is divided into interest propagation, gradient establishment and path to reinforce. Energy savings can be achieved by diffusion if empirical selection of good paths is done i.e. by processing data innetwork and by caching. This energy savings helps in achieving multipath interactions locally which is a unique feature of diffusion and improving bandwidth utilization. Directed Diffusion is not good for applications like environmental monitoring because it is a query-driven on demand data model and monitoring requires continuous data delivery to the sink. Also it is not suitable for large-scale or frequently topology changing networks.

\section{e) Fermat Point Based Energy Efficient Geocast Routing}

Geocast routing protocol is used to transfer the packets to a group of nodes that are within a particular geographical area. In reducing the energy consumption of WASN, fermat point based protocols in a multi-sink multi-hop are used which considerably reduces the total transmission distance. Congested environment in WASN may increase the multipath propagation which in turn may lead to multipath fading. The effects of both the factors are considered in [9] on the performance of I-Min [9] routing protocol designed for WASNs. I-MIN is an energy efficient scheme because the node with higher residual energy is selected even if the distance from destination is more than that for another node with a less value of residual energy. Radio model modifications with propagation environmental changes may affect the multipath fading and energy consumption in a geocast routing protocol [7]. As the number of geocast regions increases, the total distance a data packet travels also increases. Thereby, the larger is the effect of propagation environment when combined with the effect of multipath fading on the performance of an energy aware algorithm.

\section{f) Gradient-Based Routing}

Gradient Based Routing (GBR) is a reserved local predictive paradigm where each node calculates the height of the node. It is a reserved protocol as it assigns a local threshold value to each node. If the energy of a resource drops below the threshold, the height is increased so that sensors don't send data. Although an effect on delay is introduced, yet it conserves the total communication energy by balancing the distribution of network traffic. The difference between the node's height and its neighbor is called link gradient. Thus the algorithm makes an improvement to DD, in order to get the total minimum hop numbers. Traditionally, hop count was the only metric considered but now remaining energy is also used for each node while relaying data from source to sink[8]. This scheme is useful in a network with frequent topology change occurring due to node failures.

\section{g)Two-Tier Data Dissemination}

The two-tier data dissemination(TTDD) is a data-centric protocol which supports mobile sink nodes[11]. The sensors set up a grid network topology where the event message is sent to the grid. Mobile sink nodes sends query to the event associated which is broadcasted in the local area. Thus it can form a full path from the sink node to the event area. The density of nodes should be high. The TTDD is based on the transparent transmission which simplifies the maintenance and management of the network. TTDD adopts a single path mode in comparison to DD and reduces the data traffic Thereby, improving the network lifetime. But, the cost of computing and maintaining the grid network is large. 


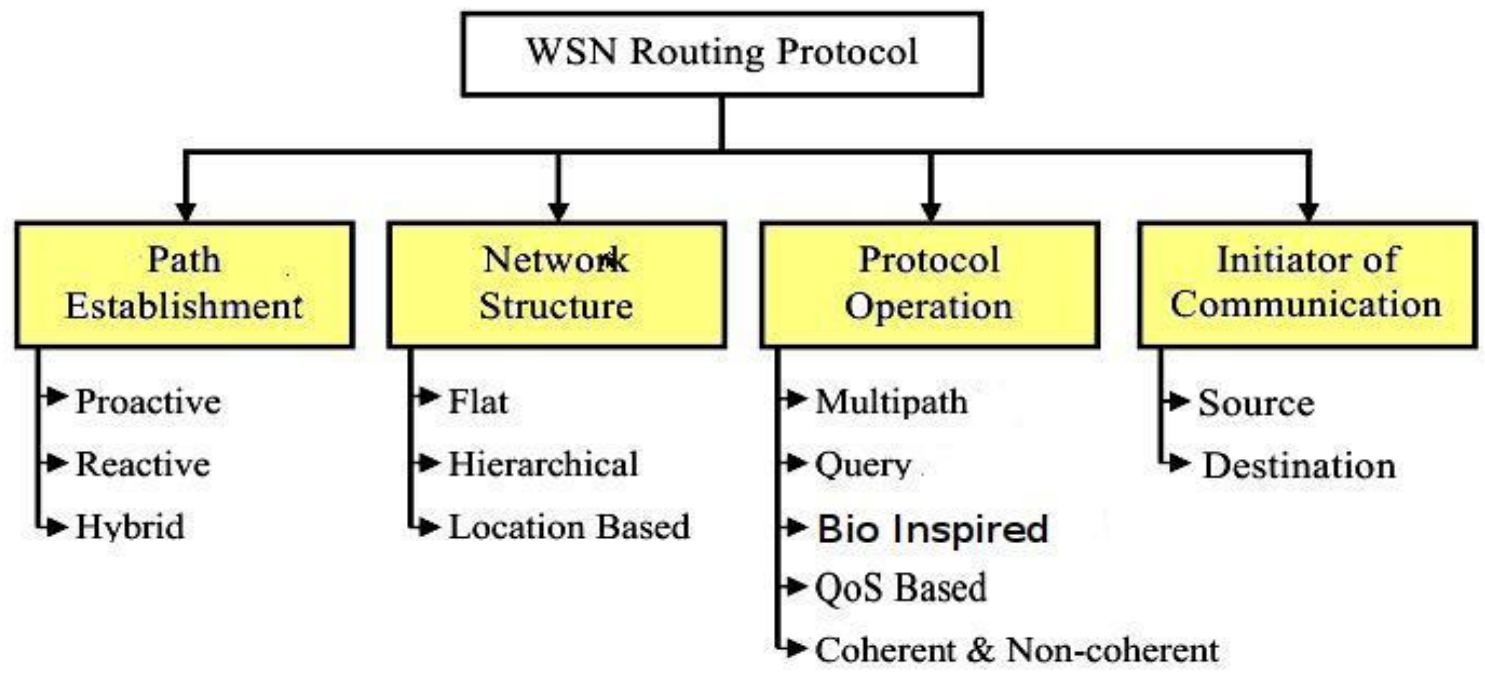

Fig 1: A Taxonomy of Wireless Sensor Network Routing Protocols

\subsubsection{Hierarchical Routing (Clustering)}

To overcome the load in carrying out long haul communication, network clustering has been pursued in some routing approaches which has led to the large coverage area without degrading the service. Hierarchical routing generally works in two layers. In the first layer cluster-head is chosen and in the second layer routing is done. These clusters perform data aggregation and fusion tasks to make WSNs more scalable and energy efficient.

\section{a) LEACH Protocol}

Low-Energy Adaptive Clustering Hierarchy (LEACH) is the first hierarchical based protocol that uses randomized rotation of local cluster base stations(BS). LEACH is used when a node in the network fails or the battery stops working. It is a self-organizing and an adaptive clustering protocol where nodes are divided into clusters and each cluster consists of Cluster Head $(\mathrm{CH})$ and a Cluster Member(CM). The $\mathrm{CHs}$ are not selected statically in a network as the sensor nodes may die quickly. LEACH uses randomized protocol to balance the energy consumption for the nodes by dividing the Cluster heads role to other nodes. Furthermore, to regulate the channel access in a cluster LEACH uses TDMA(Time Division Multiple Access) protocol[12]. $\mathrm{CHs}$ have the responsibility to assign TDMA slots to the cluster members. $\mathrm{CH}$ and $\mathrm{CM}$ communicate peer-to-peer during the time slot that has been given to that member while other members are in a sleep state resulting in a decrease in energy dissipation .

\section{b) $E-L E A C H$}

Energy-LEACH protocol(E-LEACH) improvises the $\mathrm{CH}$ selection procedure. It is divided into rounds as in LEACH. The probability for a node to be a $\mathrm{CH}$ is same in the first round. However, after this the remaining energy of every node is accounted and the node with the higher residual energy is chosen to be $\mathrm{CH}$ rather than that with lesser energy [12].

\section{c) $T L-L E A C H$}

Two-Level Hierarchy LEACH (TL-LEACH) is responsible for sending data to the base station in one hop by $\mathrm{CH}$. It consists of two level cluster heads i.e. primary and secondary. The advantage of two-level structure is that it reduces the nodes that transfers information to the BS thereby reducing the total energy usage[17].

\section{d) $M-L E A C H$}

In Multi-hop LEACH (M-LEACH) protocol, the data sent to the sink is by using a $\mathrm{CH}$ which uses the other $\mathrm{CHs}$ as relay stations [14]. The problem of distant $\mathrm{CHs}$ from the base station is solved, but they may consume large energy during the transmission of data

\section{e) $\mathrm{LEACH}-\mathrm{C}$}

Centralized LEACH(C-LEACH) protocol has no idea of $\mathrm{CH}$ and it may result in better performance by administering the cluster heads in the whole network. Here, each node sends its remaining energy to the sink and also its location. The centralized cluster algorithm is then run by the sink to know the clusters for that round. As location information is required for all the sensors it is not considered robust [15].

\section{f) $V$-LEACH}

New Version LEACH(V-LEACH) protocol has a vice-CH in a cluster in addition to a $\mathrm{CH}$ which takes the role of the $\mathrm{CH}$ when a $\mathrm{CH}$ dies [16]. Clusters are of no use if a $\mathrm{CH}$ dies because the collected information will not reach its destination.

\section{g) $U-L E A C H$}

U-LEACH merges the features of I-LEACH [23] and PEGASIS. It utilizes the multi-hop transmission approach to BS from PEGASIS and energy aware $\mathrm{CH}$ selection approach from I-LEACH. Here, Master $\mathrm{CH}(\mathrm{MCH})$ is responsible to send gathered data to BS. The clustering approach is used for selection of $\mathrm{CH}$ in comparison to probabilistic approach which earlier LEACH employed.

\section{h) PEGASIS and Hierarchical-PEGASIS}

Power-Efficient Gathering in Sensor Information Systems (PEGASIS)[25] is a chain based protocol which uses greedy algorithm to form chains like clusters in LEACH clustering. If a node fails, chain is reconstructed such that it avoids the dead node. Each node communicates to the closest neighbor and turn by turn transmit data to the base station. This results in less amount of energy spent per round. Data is gathered in each round where each node receives data from one neighbor, 
aggregates it and transmits to another neighbor in the chain. In comparison to LEACH, PEGASIS performs better as it eliminates the dynamic cluster overhead but due to asynchronous transmission time is prolonged. So it is not used for real-time application environment. PEGASIS is modified by allowing concurrent transmissions to occur when the nodes are not adjacent. This is known as Hierarchical-PEGASIS. Although these two algorithms eliminate the overhead of clusters, but they do not consider the energy of next hop while choosing a routing path. So they are not useful for heavyloaded network. Due to large delay in data transmission it is not suitable for networks where global knowledge is not available.

\section{i) $C H I R O N$}

CHIRON [22] is an energy-efficient chain based hierarchical routing protocol and is an improvement over PEGASIS. The beam Star concept [22] is applied in this algorithm which divides the sensing area in fan-shaped groups. CHIRON divides the sensing field in a number of small areas which creates multiple short chains to reduce the redundant path and transmission delay. Therefore it conserves the energy of a node and prolongs the network lifetime. Unlike traditional approaches, the sensor nodes of each group are self- organized as a chain for data dissemination. In CHIRON every node gets its location information passively from the BS with a minimum control overhead. It reports the data to the BS in a multi-hop manner. This algorithm consists of four phases namely, group construction, chain formation, leader node election and data collection and transmission phase.

\section{j) TEEN and APTEEN}

Threshold sensitive Energy Efficient(TEEN) sensor network protocol has been developed for reactive networks [24] which achieves energy saving through inhibiting unnecessary communication. It is useful for temperature sensing applications. TEEN is based on hierarchical grouping and uses LEACH clustering strategy. It divides the sensor nodes to detect the changes in the sensed environment. After the formation of clusters, TEEN separates Cluster Head and uses hard and soft thresholds to detect the sudden changes. This helps in reduction of transmission and improves energy utilization ratio and lifetime of the network. Thus it responds to real time applications and emergencies quickly but not on periodic basis making it a drawback. TDMA scheduling of nodes is done to avoid collision. But this causes delay in the reporting of time-critical data. Another possible solution is to use CDMA which is suited for the time critical applications like explosion detection, intrusion detection etc. An extension to TEEN is Adaptive Threshold sensitive Energy Efficient sensor Network protocol (APTEEN) that aims at recording of periodic data collections and reacting time of critical events. The architecture is similar to TEEN. In APTEEN after the $\mathrm{CHs}$ decision takes place, it broadcasts the parameters like count time, attributes and threshold to all nodes [25]. APTEEN's performance lies between LEACH and TEEN in terms of network lifetime and energy consumption. It makes an improvement over TEEN as it uses periodic report for the time critical events. The main drawback of these two algorithms is overhead and complexity of forming clusters.

\section{k) WB-TEEN and WBM-TEEN}

These two protocols adopt time-driven model and use distributed clustering[21]. They are basically improvements of LEACH and TEEN protocol. In LEACH there are homogeneous nodes where routing is done in a single hop via the cluster-heads $(\mathrm{CH})$ which consumes considerable amount of transmission energy. Also in TEEN, unequal number of nodes occur in different cluster making it a problem. WBTEEN tries to solve the problem by distributing an equal number of nodes in each cluster and calculates degree. On the basis of this degree it selects or rejects the membership of the node. WBM-TEEN is another protocol that not only improves the WB-TEEN but also emphasizes multi-hop intra cluster transmission of data to the sink. Performance metric measured are energy consumption with respect to the number of rounds, network lifetime of system and number of nodes alive per round.

\section{l) $H E E R P$}

Hierarchical Energy Efficient Routing Protocol(HEERP)[18] for WSN introduces a new centralized approach to hierarchy formation. It does not consider the cluster formation and cluster head selection. The algorithm generally involves network hierarchy, neighbor table construction and data transmission. Here, the sink node initiates the formation of hierarchy by broadcasting LCREQ packet. By the meantime, nodes left selects LCREQ packets from nodes with less hop counts. Therefore, avoiding flooding of packets until the construction of the network. In data transmission phase, each node sends data to their parent nodes. Node failure or battery exhaustion like factors are considered in the maintenance phase. HEERP consumes less energy than the LEACH Protocol. Also simulation results show that delay in packet delivery is improved in HEERP in comparison to LEACH .

\section{m) $H S E P$}

Heterogeneity aware Hierarchical Stable Election Protocol (HSEP) for WSNs [19] is a cluster based algorithm that shows an improvement over SEP [20]. In SEP, heterogeneity of nodes is considered which evenly consumes the extra amount of energy for advance nodes. Thus providing long stability than LEACH. Separate threshold values for advanced and normal nodes are observed. HSEP is a heterogeneous-aware clustering protocol that uses two types of $\mathrm{CHs}$ i.e. primary and secondary. The probabilities of nodes to become $\mathrm{CHs}$ is selected by initial node energy that is relative to other network nodes. This approach provides a stability period. In HSEP, primary $\mathrm{CHs}$ check the distance among themselves and transmit the data to the secondary $\mathrm{CHs}$ (which are at minimum distance). TDMA approach is used for data transmission of nodes and the primary $\mathrm{CHs}$. And also for primary and secondary CHs transmission. This improvement of HESP over SEP is due to two level hierarchy introduction.

\subsubsection{Location Routing(Geographic)}

The location information is used to locate the position of sensor nodes. To calculate the nearest neighboring node distance two techniques are used i.e. finding the coordinate of the neighboring node and the other is to use GPS (Global Positioning System). Since, sensor networks are spatially deployed in a region with no addressing scheme, location information can be utilized in routing of data in an energy efficient way.

\section{a) MECN and SMECN}

Minimum Energy Communication Network (MECN) uses low power GPS devices and sensor nodes to set up and maintain minimum energy network [26]. It finds the smallest network with minimum nodes which needs less transmission power for any two nodes(shortest path). The master - node is assumed as the information sink that develops a minimum power topology for every node. Relay region is identified for each node which helps in more energy-efficiency than with direct transmission. With the help of GPS devices optimal 
links are computed i.e. on the position coordinates basis. Moreover it dynamically adapts to deployment of new sensors and elimination of nodes as it is capable of self-reconfiguring. MECN has a drawback that it assumes that every node transmits to other nodes which may not be true always. So an extension of MECN was taken in account i.e. Small Minimum Energy Communication Network (SMECN) [27]. SMECN considers obstacles among pairs of nodes making its usage advantageous. Experimentally it is proved that SMECN uses less energy than MECN. Also the maintenance cost of the links is less. However, to find a sub-network with small edge results in more overhead of the algorithm.

\section{b) GEAR}

Geographic Energy Aware Routing(GEAR) uses energy aware heuristics and geographic information for neighbor selection which routes a packet towards the target region [28]. It aims at reducing the number of interests used in DD by adding geographic information to the interest packet. GEAR considers a particular region to send the interest to the whole network by using flooding mechanism. This way energy consumption is balanced and network lifetime is increased. If the destination node is near it uses a next hop method and in far off nodes holes occurs in GEAR. Here it forwards the packet by selecting a next-hop node which minimizes the cost value of the neighbor. Recursive Geographic Forwarding (RGEAR) algorithm disseminates the packet inside a region. In comparison to GPSR(non-energy aware routing protocol) GEAR behaves well in uneven traffic conditions. Simulation results show that GEAR receives $70 \%$ to $80 \%$ more packets than GPSR. And in the case of uniform traffic it delivers $25 \%$ to $35 \%$ more packets than GPSR.

\section{c) GAF and $H G A F$}

Geographic Adaptive Fidelity (GAF) [29] is a location-based energy-aware routing algorithm. It has been primarily designed for adhoc networks and is applicable for sensor networks. It conserves energy by not using unnecessary nodes in network as only selected messages are transmitted while others are asleep. Thus, reducing the number of nodes to form the network and saving the battery of nodes. Hierarchical Geographical Adaptive Fidelity (HGAF) also saves battery by increasing the cell of GAF. This is done by adding a layered structure in each cell. For the guaranteed connectivity in two adjacent cells, active nodes are required to make it a limitation of GAF. HGAF limits the position of active node in a cell and synchronizes the position in each cell among all cells. HGAF outperforms GAF in terms of the packet delivery ratio and survived nodes with higher node density. For randomly and dense distributed networks the lifetime of HGAF is about $200 \%$ more than GAF.

\section{d) GPSR}

Greedy Perimeter Stateless Routing (GPSR) is a geographic routing protocol where nodes forward packet locally in accordance greedy algorithm [30]. It performs well in normal circumstances. But with the obstacle introduction or inadequate sensors it may cause voids in network topology. GPSR solves this by incorporating a perimeter routing mechanism. The nodes surrounding the voids helps in their detection. Though this approach works well, other robust perimeter routing algorithm is also proposed. The graph drawn from complete network topology firstly reduces to a planar graph(where no edges crosses). Then the packet reaching a void forwards the node which in turn locates the face of planar graph. Here a node generally forwards the packet to the nodes with the edge that borders the face. e) $T B F$

Trajectory Based Forwarding (TBF) allows packets to adopt a source-specified trajectory. It is a useful paradigm for direction based routing in wireless sensor networks [31]. Unlike GPSR which sends a packet in a straight path towards the destination, TBF uses trajectory strategy. It can increase the efficiency of different forwarding protocols which includes multipath forwarding with the help of spoke broadcasting and broadcasting to a remote subregion. This helps in increasing the overall flexibility.

\subsection{Protocol Operation Routing Protocols}

On the basis of operations performed in a network-structure, protocols can be divided into multi-path, query-based, negotiation-based, quality-of-service (QoS) based, bio inspired and coherent-based routing protocols.

\subsubsection{Bio Inspired Routing}

Recently insect sensory systems are becoming an inspiration for computing and communications paradigms. This has lead to significant advancement in routing [32] like Bio Inspired routing. For instance, in Ant Colony Optimization (ACO) colony of artificial ants provides solutions. These are guided by the pheromone trails and heuristic information [33][34]. The Many-to-One-Improved Adaptive (MOIA) routing protocol is an ant colony-based routing protocol. It is coupled with a lightweight congestion control algorithm which helps in reducing the collision. Swarm intelligence and ACO [35] have been employed to find the shortest best route within a multihop WSN. Here every node has the knowledge of its location and destination. To find the best next hop to reach its destination ant-routing algorithm is discussed in detail in [36].

\section{a) MADFT}

Minimum Ant-based Data Fusion Tree(MADFT) [37] is also a routing algorithm which is based on ACO. It is used for gathering the correlated data in sensor networks. Here ants are assigned as the source nodes where the route constructed by one of the ants is followed by the other ants to search the nearest point. For its computation, the formula chosen is Probability function which is composed of pheromones and the cost to find the minimum total cost path. MADFT optimizes the transmission and fusion costs. It adopts the ant colony mechanism to achieve optimal solutions [37].

\section{b) $\mathrm{SIO}$}

Swarm Intelligence Optimization (SIO)[38] based routing algorithm balances the energy consumption globally. Some nodes may exhaust their energy early due to high concentrated routes among the nodes. The algorithm selects its next hop with the less pheromone. It differs from traditional ant colony algorithms and is better than Directed Diffusion routing protocol when end-to-end delay and energy balance factors are concerned. Also it prolongs the network lifetime due to its global energy balancing nature.

\section{c) LGossiping Protocol}

In Location based Gossiping(LGossiping) [39] protocol a node randomly chooses its neighbor with the specified transmission radius to transmit the event data. As soon as the neighbor node sees this event, it randomly selects another node with the specified transmission radius and transmits the event. This process would continue till the sink node is reached resulting in less delay than gossiping. 


\subsubsection{Query Routing}

Sensor networks are considered as a distributed database system that proposes an interface for the applications of sensor network through an SQL-like querying language. Here routing propagates the use of queries issued by the BS.

\section{a) $T A G$}

The Tiny Aggregation Service (TAG) minimizes the amount of messages transmitted during the execution of a query [40]. In standard database query execution, central processor gathers the data when a query gets executed. But TAG executes the query in a distributed fashion and reduces the amount of traffic transmitted in a network. Here time is divided into epochs for queries are returned at multiple times. When the root node sends the query, the receiving nodes set their parents as the sending node. It establishes an interval within epoch where eventual children send their aggregates.

\section{b) $A C Q P$}

Acquisitional Query Processing (ACQP) runs on a processing engine TinyDB [41] which provides a user friendly generic interface through an enhanced SQL-like interface. It enables the query execution to be optimized at many levels. ACQP follows storage points where the sensor data are created for the queries of data streams. These storage points are beneficial for sliding window type queries. Along with this, ACQP supports queries that occurs with specific events to meet certain required lifetime queries. Also ACQP provides optimization of scheduling of sensing tasks thereby minimizing the expected power consumption. Semantic Routing Trees (SRTs) are used by TinyDB where the parent node construction depends on predicates of a query for which the tree is being built.

\subsubsection{QoS Routing}

In order to maintain Quality of Service in a network, both energy and quality are to be balanced. QoS metrics which are considered during the transmission are latency, energy, bandwidth etc. while transferring data to the base Station.

\section{a) $B S A$}

Breath-a Self-Adapting (BSA) Protocol for WSNs is used in control and automation[42]. It is a novel cross layer approach that utilizes the real time control in WSNs with randomized routing. With the help of duty-cycling the consumption of energy in the network is minimized. The protocol is simple to implement, robust to use and fault tolerant. Due to randomized routing it can adapt well to the variations of traffic. The protocol generally considers physical layer and routing aspects along with randomized MAC. BSA performs better than IEEE 802.15.4 in terms of energy consumption and reliability. Thus making it scalable with large number of nodes and efficient for load balancing.

\section{b) MERR and AMERR}

Minimum Energy Relay Routing (MERR) and Adaptive MERR (AMERR) routing protocols effectively use energy with good scalability. They are less complex and focus on optimal power consumption. It is assumed that it uses a Poisson model for distribution of nodes that adopts a linear path. While AMERR is suitable for practical deployment environments, MERR performs optimally for densely deployed sensor networks. While MERR assumes that sensor nodes are aware of the distances of downstream neighbors, AMERR takes into account the distance to the base station. Thus making a better routing decision than MERR [43].

\section{c) $S A R$}

Sequential assignment routing (SAR) protocol is the first protocol to introduce QoS routing decisions [44]. It is a table driven multipath protocol that strives for energy efficiency and fault tolerance. It creates tree with one-hop neighbors by considering QoS metric, priority level and energy resource on every path. Created trees are used to form multiple paths from sink to sensors. According to QoS and energy resources one of these paths is selected. Due to the routing table consistency between upstream and downstream nodes for each path node failure problem is recovered. SAR offers lower power consumption than minimum-energy metric algorithm as it focuses on the energy consumption and not on packets priority. SAR can maintain multiple paths from the nodes to the sink. Thus, making it fault tolerant.

\subsubsection{Multipath routing}

Multipath protocols are efficient in maintaining multiple paths between source and destination. If the primary path fails in a network, alternate paths are chosen resulting in reliability and fault tolerance of the protocol. Due to which overhead cost and energy consumption cost may increase.

\section{a) $Q E M P R$}

By using the link quantity as a performance metric the authors in [45] propose a multipath routing protocol, QoS and energyaware multipath routing (QEMPR) for real-time application in WSNs. Each node in the network is assigned with a unique ID and also has a capability of calculating the packet receiving and packet sending probability using the link quality information. The multiple paths are discovered by message broadcasting and each node maintains a neighboring table which stores the information about the neighboring node such as remaining energy and transmission range. After constructing the paths, the packet will be transmitted based on the packet sequence number and number of hops it is away from the sink. This approach helps to distribute the network traffic throughout the multiple paths. Thus increasing network lifetime.

\section{b) EEAMR}

In [46], the authors propose EEAMR which focuses on distributing the traffic based on the node's residual energy and received signal strength. For consistent resource utilization, more load is assigned to under-utilized paths and less load is assigned to over-utilized path. In order to save more energy, nodes which are not participating in the data transmission go into sleep mode.

\section{c) REEM}

In Reliable and Energy Efficient multipath (REEM) [47]routing protocol multiple paths are constructed from source to destination, considering node reliability and energy level. The path is constructed by a base station through message broadcasting and each receiving node will store the neighboring information in a table. Also, the path reliability is evaluated by the base station through a weighted and oriented graph, based on the neighbor information.

\section{d) $M R M S$}

It is more challenging to be energy-efficient in large scale sensor networks. So to overcome this in [48] authors have proposed a novel protocol named Multipath Routing with Multiple sink nodes(MRMS) in large scale sensor networks to save energy. The main idea is to deploy multiple sink nodes and uses path cost metric to select the multiple paths. The path cost metric is defined based on the distance between two neighbors, hop count and available energy at the node. In 
some scenarios the WSN is deployed in such an environment where the base station needs to query a certain portion of the network to collect the sensing information from the nodes.

\section{e) $E B M R$}

Energy balancing multipath routing(EBMR) protocol [49] is based on a client - server architecture with the base station processing the data received from the sensor nodes. The path construction is done by using message broadcasting from the base station. Each node in the network contains a neighboring table. Whenever the base station needs to query some data in the network it broadcasts a Data Enquiry (DE) message. The nodes which have the required data will reply back with a Data Enquiry Reply (DER) message. Upon receiving the DER message, the base station will calculate the shortest path to the source node by calculating the amount of energy consumes in transmitting the package from source to base station.

\subsubsection{Coherent and non-coherent protocols}

Data processing is an important event in the operation of wireless sensors which employs two different routing techniques within a network i.e. coherent and non-coherent.

\subsubsection{Non-coherent data Routing}

In this routing, nodes (aggregators) that perform processing will first collect and process the raw data before sending to further nodes for processing.

\section{a) $S W E$}

Single winner algorithm (SWE)[50] has a single aggregator node which is selected for complex data processing. The node selection is based on nodes computational capability and energy reserves. So whenever the SWE process ends, a minimum hop spanning tree is completely covered.

\subsubsection{Coherent data Routing}

In this routing, after minimum processing (like time stamping, duplicate suppression) data is forwarded to the aggregators. It can be considered energy efficient if the less number of senders send data to the aggregator or central node.

\section{a) $M W E$}

Multiple winner algorithm (MWE)[50] is an extension to SWE. When nodes act as sources and start sending their data to the central node(aggregator), a large amount of energy consumption occurs leading to high cost. So to lower the energy cost the sources are limited, which sends data to the central node. Here each node keeps a record of n nodes. Thus, MWE results in more delay, larger overhead and less scalability when compared to non-coherent protocol.

\subsection{Established path based routing protocol}

On the basis of communication paths followed by a node, the protocols in the network are classified as proactive, reactive and hybrid. Due to thousands of nodes employed in WSNs it becomes difficult to manage routing tables. Therefore, proactive protocols are not suited to WSNs.

\subsection{Initiator of Communication}

On the basis of application whether a source or sink wants to initiate the communication or transmission of packets this routing protocol is classified.

\section{CONCLUSION}

Sensor network routing has gained large attention in the recent years. In comparison to traditional data routing in wired networks it has introduced unique challenges. This paper presents a comprehensive survey of the routing techniques for
WSNs from the recent work. The main categories explored in this paper are bio-inspired routing protocols, QoS, hierarchical based and location-based routing. Each routing protocol is discussed under the appropriate category. In future research, focus on sensor network routing protocols and its integration with wired networks would be considered. Applications used for security and environmental monitoring mostly requires data collection from the sensor nodes to be transmitted to a server so that further analysis can be done. As the routing requirements of every environment are different, further research can be carried out considering such situations.

\section{REFERENCES}

[1] Alaauldin Ibrahim,Malik Kemal Sis and Sen Cakir," Integrated Comparison of Energy Efficient Routing Protocols in Wireless Sensor Network: A survey" 2011 IEEE Symposium on Business, Engineering and Industrial Applications (ISBEIA), Langkawi, Malaysia.

[2] Prathap U, Deepa Shenoy P and Venugopal K R," Wireless Sensor Networks Applications and Routing Protocols: Survey and Research Challenges" 2012 International Symposium on Cloud and Services Computing, DOI 10.1109/ISCOS.2012.21

[3] J. S. Wilson, Sensor technology handbook. USA: Elsevier. 2005

[4] Joanna Kulik, Hari Balakrishnan and W. R. Heinzelman, (1999) " Adaptive Protocols for Information Dissemination in Wireless Sensor Networks", Proceedings on the 5th annual ACM/IEEE international conference on Mobile computing and networking, pp. 174-185.

[5] Edward Woodrow and Wendi Heinzelman , (2002) "SPIN-IT: A Data Centric Routing Protocol for Image Retrieval in Wireless Networks",ICIP (3), pp. 913-916.

[6] D. B. a. D. Estrin., "Rumor routing algorthim for sensor networks.," in 1st ACM international workshop on Wireless sensor networks and applications, WSNA,2002.

[7] Chalermek Intanagonwiwat, Ramesh Govindan, Deborah Estrin, John Heidemann and Fabio Silva, "Directed Diffusion for Wireless Sensor Networking", IEEE/ACM Transactions on Networking (TON), vol.11, pp. 2-16, February 2003.

[8] Li Xi Chen and Xiaohong Guan, (2004) "A New GradientBased Routing Protocol in Wireless Sensor Networks", Proceedings of the First international conference on Embedded Software and Systems, PP. 318-325.

[9] Kaushik Ghosh, Partha Pratim Bhattacharya and Pradip K Das, "Effect of multipath fading and propogation environment on the performance of a fermat point based energy efficient geocast routing protocol", International Journal of Wireless \& Mobile Networks (IJWMN), Vol. 4, No. 1, February 2012

[10] Ankita Joshi \& Lakshmi Priya.M, "A Survey of Hierarchical Routing Protocols in Wireless Sensor Network", MES Journal of Technology and Management, pp. $67-71$.

[11] K. Sohrabi et al., Protocols for self-organization of a wireless sensor network, IEEE Personal Communications pp. 16-27, May 2000. 
[12] F. Xiangning, S. Yulin. "Improvement on LEACH Protocol of Wireless Sensor Network", 2007, International Conference on Sensor Technologies and Applications pp 260-264, ido:10.1109/SENSORCOMM.2007.60

[13] S. Tilak et al., A taxonomy of wireless microsensor network models, Mobile Computing and Communications Review 6 (2) (2002) 28-36.

[14] Dissertation, H. Zhou, Z. Jiang and M. Xiaoyan, "Study and design on cluster routing protocols of wireless sensor networks",2006.[15] W.B. Heinzelman, A.P. Chandrakasan, H. Balakrishnan, "An application-specific protocol architecture for wireless microsensor networks," IEEE transactions on wireless communications Vol. 1, NO. 4, pp. 660-670, Oct. 2002

[16] M.B. Yassein, A. Al-zou'bi, Y. Khamayseh and W. Mardini, "Improvement on LEACH protocol of wireless sensor network (VLEACH)". International Journal of Digital Content Technology and its Applications. Vol.3, No.2, pp. 132-136 Jun 2009

[17] V. Loscrì, G. Morabito and S. Marano. "A Two-Levels Hierarchy for Low-Energy Adaptive Clustering Hierarchy".

[18] Ming Zhang, Yanhong Lu, Chenglong Gong, “ EnergyEfficient Routing Protocol based on Clustering and Least Spanning Tree in Wireless Sensor Networks", International Conference on Computer Science and Software Engineering,IEEE, 2008

[19] A. A. Khan, N. Javaid, U. Qasim , Z. Lu , Z. A. Khan ,'HSEP: Heterogeneity-aware Hierarchical Stable Election Protocol for WSNs "Seventh International Conference on Broadband, Wireless Computing, Communication and Applications", 978-0-7695-48425/12 2012 IEEE.

[20] Smaragdakis, G. and Matta, I. and Bestavros, A., "SEP: A stable election protocol for clustered heterogeneous wireless sensor networks", Boston University Computer Science Department, 2004.

[21] Zibouda Aliouat, Saad Harous.” An Efficient Clustering Protocol IncreasingWireless Sensor Networks Life Time", International Conference on Innovations in Information Technology (IIT),2012 IEEE

[22] Kuong-Ho Chen, Jyh-Ming Huang, Chieh-Chuan Hsiao, "CHIRON: An Energy- Efficient Chain-Based Hierarchical Routing Protocol in Wireless Sensor Networks", IEEE

[23] Naveen Kumar, Sandeep, Pawan Bhutani, Prity Mishra ,'U-LEACH: A Novel Routing Protocol for Heterogeneous Wireless Sensor Networks" 2012 International Conference on Communication, Information \& Computing Technology (ICCICT), Oct. 19-20, Mumbai, India.

[24] Arati Manjeshwar and Dharma P. Agrawal, "TEEN: A Routing Protocol for Enhanced Efficiency in Wireless Sensor Networks", Parallel and Distributed Processing Symposium,proceedings 15th International, pp. 20092015, April. 2009.

[25] Arati Manjeshwar and Dharma P. Agrawal, "APTEEN: A Hybrid Protocol for Efficient Routing and Comprehensive Information Retrieval in Wireless Sensor
Networks"Parallel and Distributed Processing Symposium, proceedings International, IPDPS 2002, Abstracts, and CD-ROM, PP. 195-202.

[25]S. Lindsey, C.S. Raghavendra, "PEGASIS: power efficient gathering in sensor information systems", in Proceedings of the IEEE Aerospace Conference, Big Sky, Montana, March 2002, vol. 3.

[26] Xu, N. (2002). A survey of sensor network applications, IEEE Communications Magazine 40.

[27] L. Li, J.Y. Halpern. "Minimum-Energy Mobile Wireless Networks Revisited". IEEE International Conference on Communications. Vol. 1. 2001. pp. 278-283.

[28] Y. Yu, D. Estrin, and R. Govindan, "Geographical and energy aware routing: a recursive data dissemination protocol for wireless sensor networks", UCLA Computer Science Department Technical Report, UCLA-CSD TR01-0023,May. 2001.

[29] Tokuya Inagaki and Susumu Ishihara, "HGAF: A power saving scheme for wireless sensor network", Journal of Information Processing, vol. 17, pp. 255- 266, Oct. 2009.

[30] B. Karp and H. T. Kung, "GPSR: Greedy perimeter stateless routing for wireless sensor networks", in the Proceedings of the 6th Annual ACM/IEEE International Conference on Mobile Computing and Networking (MobiCom '00), Boston, MA, August 2000.

[31]D. Niculescu and B. Nath. Trajectory based forwarding and its applications. In Proceedings of the Ninth Annual International Conference on Mobile Computing and Networking (MobiCom), 2003.

[32] Z. (Sam) Ma, A.W. Krings, Insect sensory systems inspired computing and communications, Ad Hoc Netw. (2008), doi:10.1016/j.adhoc.2008.03.003

[33] Colorni A , Dorigo M , Maniezzo V. "Distributed optimization by ant colonies". Proc 1st European Conf on Artificial Life Paris, France:Elsevier Publishing, 1991, pp.134-142.

[34] Colorni A, Dorigo M, Maniezzo V. "An investigation of some properties of an ant algorithm". In Proc.PPSN '92Brussels, Belgium: Elsevier Publishing, 1992, pp.509-520.

[35] G. D. Caro, and M. Dorigo, "AntNet: Distributed Stigmergetic Control for Communications Networks," J. of Art. Intel. Research, vol. 9, Dec. 1998, pp. 317-365.

[36] R. GhasemAghaei, M. A. Rahman, W. Gueaieb, and A. El Saddik, "Ant Colony-Based Reinforcement Learning Algorithm for Routing in Wireless Sensor Networks," IEEE IMTC 2007, Warsaw, Poland, May 2007.

[37] Luo Juan, Song Chen, and Zhou Chao, "Ant System based Anycast Routing in Wireless Sensor Networks", in the proceedings of International Conference on Wireless Communications, Networking and Mobile Computing, 2007, WiCom 2007, Sept. 2007, pp. 2420-2423.

[38] Chao Wang and Qiang Lin, "Swarm intelligence optimization based routing algorithm for Wireless Sensor Networks" , in the proceedings of International Conference on Neural Networks and Signal Processing, 2008, June 2008, pp. 136-141. 
[39] S. Kheiri, MB. Ghaznavi Goushchi,M. Rafiee and B. Seyfe "An improved gossiping data distribution technique with emphasis on Reliability and Resource Constraints" IEEE 2009 International conference on communications and mobile computing, vol. 2, pp.247252,2009

[40] S. Madden, M. Franklin, J. Hellerstein, and W. Hong. TAG: a tiny aggregation service for ad-hoc sensor networks. In Proceedings of the ACM Symposium on Operating System Design and Implementation (OSDI), 2002.

[41] S. Madden, M. Franklin, J. Hellerstein, and W. Hong. The design of an acquisitional query processor for sensor networks. In Proceedings of the ACM SIGMOD International Conference on Management of Data, 2003

[42] Park, P.G., Fischione, C., Bonivento, A., Johansson, K.H. And Sangiovanni-Vincentelli, A., "Breath: A SelfAdapting Protocol for Wireless Sensor Networks in Control and Automation", Proceedings of 5th Annual IEEE Communications Society Conference on Sensor, Mesh and Ad Hoc Communications and Networks, 2008. SECON'08, On page(s): 323-331,ISBN: 978-1-42441777-3, June 2008.

[43] Marco Zimmerling, Waltenegus Dargie, Johnathan M. Reason, "Localized power-aware routing in linear wireless sensor networks" in the Proceedings of the 2nd ACM international conference on Context-awareness for self-managing systems (2008), pp. 24-33. Sydney, Australia, ISBN:978-1-60558-010-4 .

[44] K. Sohrabi, J. Pottie, "Protocols for self-organization of a wireless sensor network", IEEE Personal Communications, Volume 7, Issue 5, pp 16-27, 2000.
[45] Saeed Rasouli Heikalabad, Hossein Rasouli, Farhad Nematy, and Naeim Rahmani. Qem-par: Qos and energy aware multi-path routing algorithm for real-time applications in wireless sensor networks. CoRR, abs/1104.1031, 2011.

[46] R. Vidhyapriya and Dr. P. T. Vanathi. Energy efficient adaptive multipath routing for wireless sensor networks. Proc. of the IAENG International Journal of Computer Science, 34, 2007.

[47] Xin-hua Wang, Chang-ming Che, and Ling Li. Reliable multi-path routing protocol $\mathrm{n}$ wireless sensor networks. In Proceedings of the 2010 International Conference on Parallel and Distributed Computing, Applications and Technologies, pages 289-294, 2010.

[48] Yuequan Chen, Edward Chan, and Song Han. Energy efficient multipath routing in large scale sensor networks with multiple sink nodes. In Advanced Parallel Processing Technologies, volume 3756, pages 390-399. 2005

[49]Chen Yunfeng and Nasser Nidal. Energy-balancing multipath routing protocol for wireless sensor networks. In Proceedings of the $3 \mathrm{rd}$ international conference on Quality of service in heterogeneous wired/wireless networks, 2006.

[50] K. Sohrabi, J. Pottie, "Protocols for self-organization of a wireless sensor network", IEEE Personal Communications, Volume 7, Issue 5, pp 16-27, 2000. 